\title{
A rare case of central nervous system amyloidoma treated with fractionated radiotherapy
}

\author{
Teresa Meier, MD, ${ }^{1}$ J. Michael Hazenfield, MD, ${ }^{2}$ Saulius Girnius, MD, ${ }^{3}$ Matthew Hagen, MD, PhD, ${ }^{4}$ \\ Ronald E. Warnick, MD, ${ }^{5}$ and Jordan Kharofa, MD ${ }^{1}$
} Departments of ${ }^{1}$ Radiation Oncology, ${ }^{2}$ Radiology, ${ }^{3}$ Hematology Oncology, ${ }^{4}$ Pathology, and ${ }^{5}$ Neurosurgery, University of Cincinnati
Medical Center, Cincinnati, Ohio

\begin{abstract}
A 54-year-old female presented with multiple episodes of emesis, intractable headaches, worsening balance, and slowly progressive right facial weakness. Imaging demonstrated a 3-cm mass in the left internal capsule and corona radiata region with associated edema, mass effect, and midline shift concerning for high-grade glioma, lymphoma, or brain metastasis. Stereotactic biopsy of the mass was consistent with amyloid deposition. Systemic workup for amyloidosis was negative, and the mass was thought to represent a focal tumor-like deposit of amyloid, also referred to as "amyloidoma." In the absence of systemic disease, therapy, which can include surgery or radiotherapy, can be directed at the local process. The location of the patient's lesion was not amenable to resection; therefore, she was treated with fractionated radiotherapy of $30.6 \mathrm{~Gy}$ at $1.8 \mathrm{~Gy}$ per fraction. Serial brain MRI demonstrated stability 18 months out from therapy. To the authors' knowledge, this is the first documented case of focal fractionated radiotherapy for CNS amyloidoma. The authors concluded that radiotherapy can prevent further progression of amyloidomas in anatomical locations that prohibit resection.
\end{abstract}

https://thejns.org/doi/abs/10.3171/2016.7.JNS1690

KEY WORDS amyloidoma; radiotherapy; CNS; MRI

$66 A$ MYLOIDOSIs" refers to the extracellular tissue deposition of insoluble fibrils and is often a systemic disease in which many organs can be affected including the liver, kidneys, heart, lungs, or central nervous system (CNS). Therapy is often aimed at the underlying etiology, such as an infectious or inflammatory process or plasma cell dyscrasia, and generally requires chemotherapy. In rare cases there can be tumor-like focal deposits of amyloid, referred to as "amyloidoma." When there is no systemic disease associated with the amyloidoma, therapy, which can include surgery or radiotherapy, is often directed at the local process. Central nervous system amyloidomas are rare with only a few dozen cases reported in the literature, and although treatment data are sparse, a majority of these lesions have been treated with resection. ${ }^{3}$ While radiotherapy has been documented as a primary treatment for amyloidoma of the lung, the present case is the first reported instance of focal radiotherapy for CNS amyloidoma.

\section{Case Report}

History and Examination

A 54-year-old female presented with multiple episodes of emesis, intractable headaches, worsening balance, and slowly progressive right facial weakness. Her physical examination was only notable for right-sided facial droop. A noncontrast head CT demonstrated a 3-cm mass in the left internal capsule and corona radiata region with associated edema, mass effect, and midline shift. She was transferred to our facility for further evaluation, which included perfusion and spectroscopic MRI and fluorine-18-labeled fluorodeoxyglucose (FDG) PET-CT. She was treated with dexamethasone for the vasogenic edema with improve-

ABBREVIATIONS AL = amyloid light chain; $C$ o = choline; $C N S=$ central nervous system; $\mathrm{Cr}=$ creatine; $\mathrm{FDG}=$ fluorine-18-labeled fluorodeoxyglucose; $\mathrm{NAA}=\mathrm{N}$-acetylaspartate. 

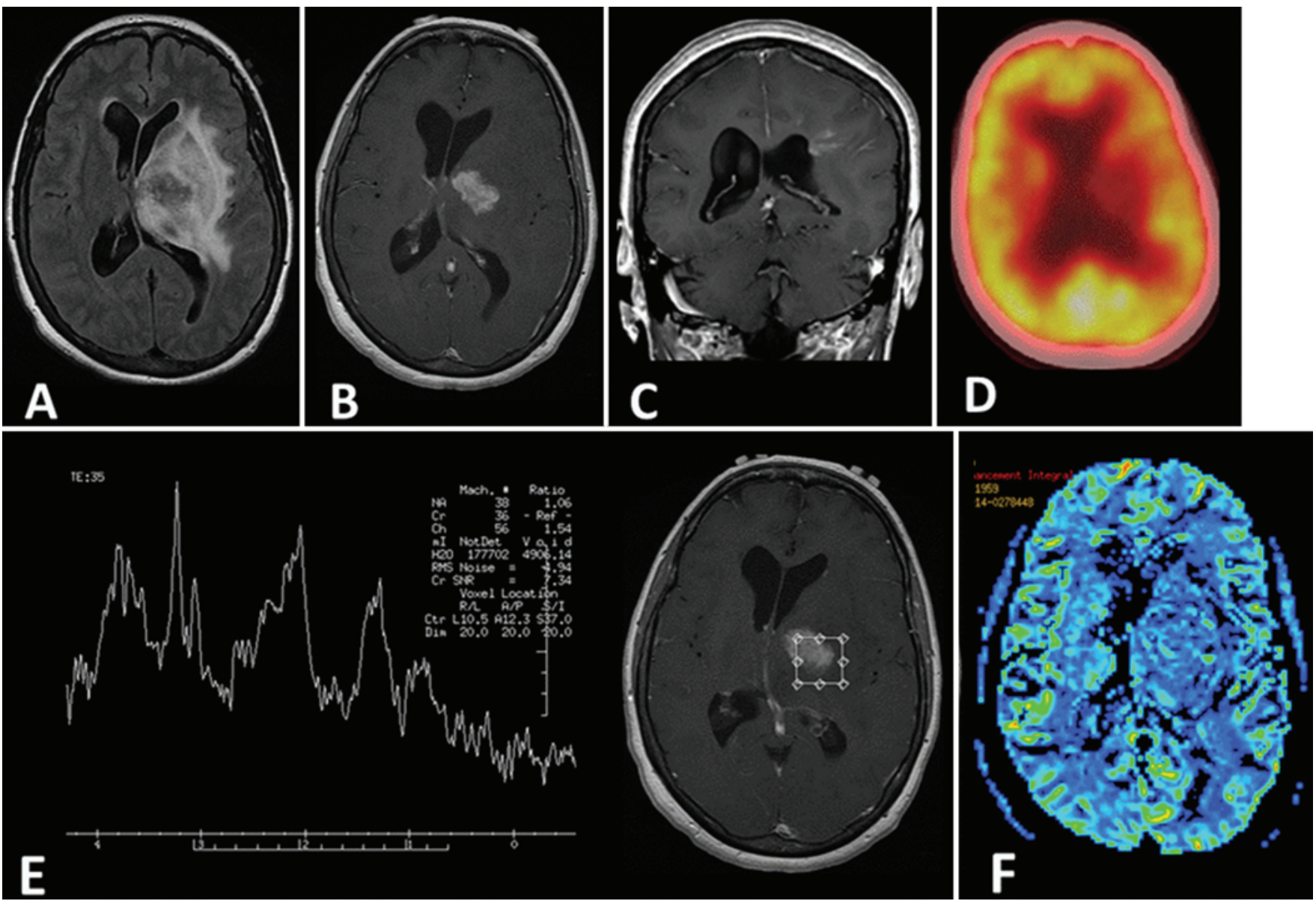

FIG. 1. Conventional and advanced imaging of the brain. Predominately hypointense T2 signal mass centered within the left internal capsule and corona radiata region with surrounding T2 FLAIR signal hyperintensity (A). There is resulting mass effect with a 10-mm left-right midline shift. After the administration of contrast there was heterogeneous enhancement (B) with radiating linear enhancement extending from the posterior aspect of the left lateral ventricle (C). A PET-CT scan showing decreased FDG uptake (D) in the region of the enhancing mass, confirming hypometabolism. Single-voxel short-echo (TE $35 \mathrm{msec}$ ) MR spectroscopy spectrum (E). Dynamic susceptibility contrast (DSC) perfusion MRI generated a cerebral blood volume color map (F). Figure is available in color online only.

ment in her symptoms. Magnetic resonance imaging demonstrated a relatively hypointense T2 signal heterogeneously enhancing mass with surrounding T2 FLAIR hyperintensity (Fig. 1A and B). We also noted a radiating linear enhancement pattern extending from the lesion periphery to the ventricular surface (Fig. 1C). On gradient imaging, patchy hypointensity thought to represent hemorrhagic changes was seen. Based on these conventional MRI findings, the leading diagnosis was glioblastoma multiforme, lymphoma, or, less likely, metastasis. However, dynamic susceptibility contrast (DSC) perfusion imaging (Fig. 1F) showed decreased relative cerebral blood volume, indicating less vasculature than the contralateral normal tissue, which is atypical for both high-grade tumors and lymphoma. Magnetic resonance spectroscopy (Fig. 1E) showed moderate to severe reduced $N$-acetylaspartate (NAA)/creatine (Cr) ratio and moderately elevated choline (Cho)/Cr ratio within the enhancing lesion. At this point the differential diagnosis was expanded to include tumefactive demyelinating disease and inflammatory mass (pseudotumor). A PET-CT scan (Fig. 1D) was also obtained, demonstrating decreased FDG uptake and no extracranial malignancy.

Image-guided stereotactic biopsy of the lesion revealed abundant, amorphous eosinophilic material with intervening small vessels and scattered mononuclear cells. A Congo red stain was consistent with amyloid, displaying apple-green birefringence under polarized light (Fig. 2). Immunohistochemical stains for $\mathrm{CD} 3$ ( $\mathrm{T}$ cell marker) and CD20 and PAX-5 (B-cell markers) revealed scattered, mature-appearing lymphocytes within the tissue. Staining for CD138 highlighted rare plasma cells. Liquid chromatography tandem mass spectrometry was performed, and the peptide profile was consistent with amyloid light chain (AL) (lambda)-type amyloid deposition.

Workup for systemic amyloidosis was unremarkable, including a normal bone marrow biopsy, serum immunofixation electrophoresis and free light chain assays, cardiac biomarkers, 24-hour urine total protein test, and echocardiogram. Repeat MRI of the head 1 month later following steroid therapy demonstrated a significant decrease in edema in the left hemisphere with improvement in mass 


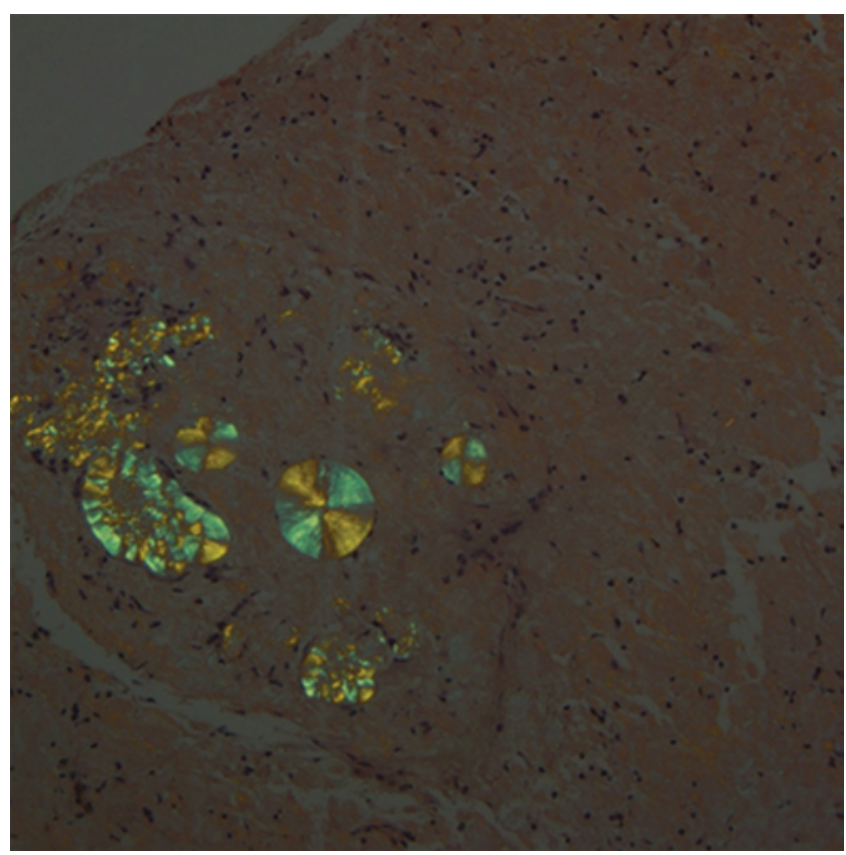

FIG. 2. Abundant amorphous congophilic material displaying applegreen birefringence under polarized light. Scattered mononuclear cells are in the background. Congo red, original magnification $\times 200$. Figure is available in color online only.

effect and midline shift; however, the irregular enhancing mass in the left corona radiata remained unchanged.

\section{Treatment}

After multidisciplinary discussion regarding potential local therapy options, the patient was referred to radiation oncology given the lack of surgical options based on the amyloidoma location. The rationale for radiation therapy was elimination of the presumed clonal plasma cell population responsible for amyloid deposition to prevent further progression and to allow for the cessation of steroid therapy. The patient was treated with fractionated radiation therapy to $30.6 \mathrm{~Gy}$ at $1.8 \mathrm{~Gy}$ per fraction to the lesion and surrounding T2 FLAIR abnormality (Fig. 3). She tolerated the treatment well with no significant difficulties. She was tapered off dexamethasone. Serial brain MRI demonstrated no progression of the edema or enhancement 18 months from the completion of therapy (Fig. 4). She still has a slight right facial droop but otherwise remains neurologically intact.

\section{Discussion}

Reported cases of amyloidoma within the CNS are rare, and treatment details for these tumors are often sparse. Central nervous system amyloidomas are typically supratentorial and subcortical and frequently affect the white matter. ${ }^{6}$ The conventional imaging characteristics can mimic those of high-grade gliomas, lymphomas, or brain metastases. Central nervous system amyloidoma on MRI often ranges from hypointense to isointense on T2-weighted images, and enhancement is typically intense but heterogeneous. ${ }^{2}$ Our case demonstrates the previously report-
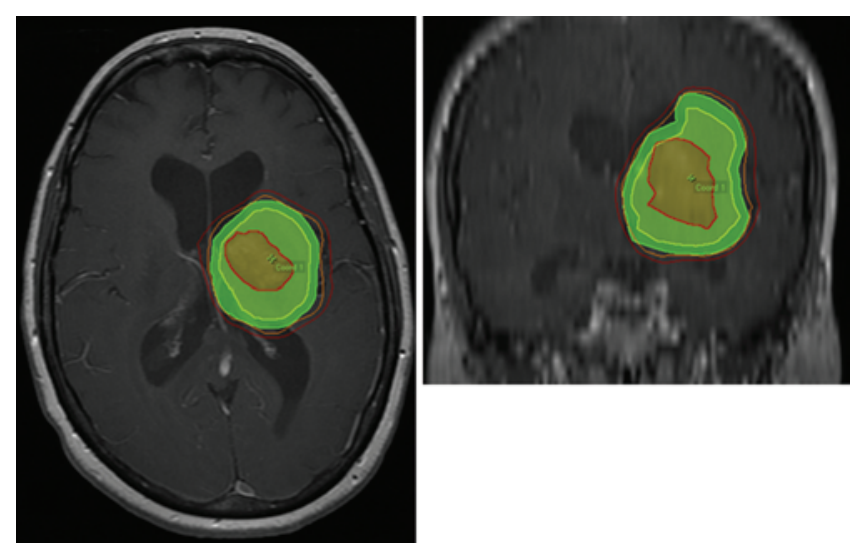

FIG. 3. Axial (left) and coronal (right) T1 postcontrast MRI of the brain. The gross tumor volume, contoured in red, includes the enhancing lesion. The clinical target volume, contoured in yellow, encompasses the enhancing component and associated T2 FLAIR abnormality. A 3-mm planning target volume, contoured in green, was added. The orange line represents the $95 \%$ isodose line, and the red line indicates the $85 \%$ isodose line. Figure is available in color online only.

ed MRI findings of extension to the ventricular surface as well as a peripheral margin of radiating linear enhancement, which can indicate deposition of amyloid along the vessels. ${ }^{5}$ Spectroscopic MRI often shows an elevated Cho/ $\mathrm{Cr}$ ratio and depressed NAA/Cr ratio, as was seen in the present case. ${ }^{9}$ The increased $\mathrm{Cho} / \mathrm{Cr}$ ratio is postulated to be the result of decreased $\mathrm{Cr}$ rather than increased Cho, the latter of which is a marker for cell membrane turnover and is elevated in high-grade tumors. ${ }^{8} \mathrm{~N}$-acetylaspartate is a neuronal marker that is often decreased with the loss of neuronal viability. Perfusion MRI will show hypoperfusion, and PET-CT will show hypometabolism, both of which are not consistent with high-grade tumors.

The presence of lambda light chain precursor proteins narrows the diagnosis to systemic rather than localized AL amyloidosis. The lack of involvement of other organs, the absence of plasma cell dyscrasia on bone marrow biopsy, and normal serum free light chains and immunofixation electrophoresis are necessary to exclude systemic AL amyloidosis. In localized AL amyloidosis, the precursor proteins are typically produced by plasma cells and occasionally from indolent B-cell neoplasms. ${ }^{4}$ In our case, rare mixed lymphocytes and plasma cells were identified. The paucity of plasma cells makes the demonstration of clonality difficult using immunohistochemical techniques. Differentiating localized from systemic AL amyloidosis is imperative since treatment of the latter requires systemic chemotherapy, whereas radiation therapy and resection can be curative in localized amyloidosis.

The clinical course of CNS amyloidoma is often benign. Resection is typically curative, although long-term follow-up has not been published. ${ }^{6}$ Lesions that have only been biopsied with no resection have shown interval growth and the potential for progression. ${ }^{3}$ Further growth of an amyloidoma in eloquent areas of the brain could lead to a loss of neurological function and worsening quality of life. Since plasma cells within the CNS represent the underlying etiology for amyloid deposition and plasma cells are known to be radiosensitive, radiotherapy was deliv- 

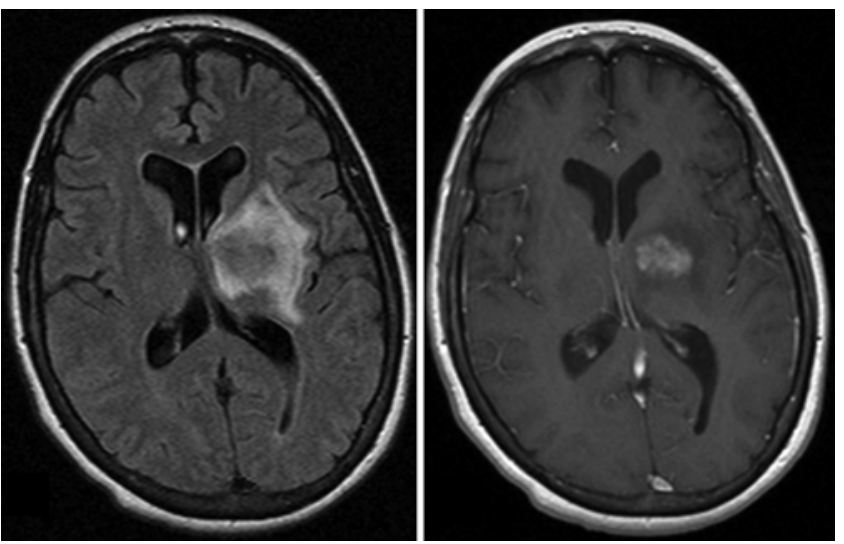

FIG. 4. Brain MRI 18 months posttherapy. Axial T2 FLAIR sequence (left) demonstrating overall decreased signal abnormality. Axial T1 postcontrast image (right) shows no evidence of progression of the enhancing lesion in the left internal capsule and corona radiata region.

ered with the intent of halting further amyloid deposition. Solitary plasmacytomas, which are tumors that form as a result of monoclonal plasma cell proliferation, are often treated with definitive radiotherapy at doses typically ranging between 30 and $45 \mathrm{~Gy}$, which effectively eliminate the neoplastic plasma cells. Therefore, it is reasonable to use similar doses to treat CNS amyloidoma to eliminate the plasma cells, which are responsible for the amyloid deposition. It is unlikely that radiotherapy will cause regression of amyloid that has already been deposited; hence, we would not expect the enhancing component of the lesion to decrease significantly on subsequent imaging but would expect to see stability of the lesion. Malignant plasma cells can secrete inflammatory cytokines and vascular endothelial growth factor, so radiotherapy may also prevent further reaccumulation of edema and thus allow patients to be tapered off steroids. ${ }^{1}$ While corticosteroids allow for a decrease in cerebral edema and improvement in neurological symptoms, corticosteroids alone would not have been sufficient in managing the amyloidoma as they do not address the underlying clonal plasma cell population that is depositing the amyloid. With no localized treatment, whether resection or radiotherapy, the plasma cells could continue to deposit amyloid and thus result in further mass effect and potential worsening of neurological symptoms. To our knowledge, there are no reported data on using steroids alone in the treatment of localized amyloidoma.

Others have reported the use of radiotherapy of $20 \mathrm{~Gy}$ in 10 fractions for amyloidoma in the lung with a complete clinical and radiographic response..$^{10}$ There is one other reported case on the use of fractionated external beam radiotherapy for primary CNS amyloidoma; however, the patient received 30 Gy of whole brain radiation since she had deteriorating mental function and a primary brain tumor was suspected based on imaging.? Follow-up imaging 6 months later demonstrated a large enhancing mass in the left parietal region without surrounding edema, and biopsy at that time revealed amyloid deposition. The patient was in good general condition 5 years later, which supports the use of relatively low doses of radiotherapy to halt the progression of an amyloid deposition. While whole brain radiation is appropriate for a patient with suspected high-grade glioma and a poor performance status, focal radiotherapy should be sufficient for primary CNS amyloidoma as local plasma cells are contributing to the amyloid deposition. We elected to include the patient's enhancing lesion in the left internal capsule and corona radiata and associated T2 FLAIR abnormality in our clinical target volume because the volume was fairly modest. While CNS amyloidoma is rare, radiotherapy should be considered to prevent further progression in anatomical locations that prohibit resection.

\section{References}

1. Anderson KC: Multiple myeloma. Advances in disease biology: therapeutic implications. Semin Hematol 38 (2 Suppl 3):6-10, 2001

2. Caerts B, Mol V, Sainte T, Wilms G, Van den Bergh V, Stessens L: CT and MRI of amyloidoma of the CNS. Eur Radiol 7:474-476, 1997

3. Fischer B, Palkovic S, Rickert C, Weckesser M, Wassmann $\mathrm{H}$ : Cerebral AL $\lambda$-amyloidoma: clinical and pathomorphological characteristics. Review of the literature and of a patient. Amyloid 14:11-19, 2007

4. Foreid H, Barroso C, Evangelista T, Campos A, Pimentel J: Intracerebral amyloidoma: case report and review of the literature. Clin Neuropathol 29:217-222, 2010

5. Gandhi D, Wee R, Goyal M: CT and MR imaging of intracerebral amyloidoma: case report and review of the literature. AJNR Am J Neuroradiol 24:519-522, 2003

6. Landau D, Avgeropoulos N, Ma J: Cerebral amyloidoma mimicking intracranial tumor: a case report. J Med Case Reports 4:308, 2010

7. Lee J, Krol G, Rosenblum M: Primary amyloidoma of the brain: CT and MR presentation. AJNR Am J Neuroradiol 16:712-714, 1995

8. Nossek E, Bashat DB, Artzi M, Rosenberg K, Lichter I, Shtern O, et al: The role of advanced MR methods in the diagnosis of cerebral amyloidoma. Amyloid 16:94-98, 2009

9. Ragel BT, Blumenthal DT, Browd SR, Salzman KL, Jensen RL: Intracerebral amyloidoma can mimic high-grade glioma on magnetic resonance imaging and spectroscopy. Arch Neurol 63:906-907, 2006

10. Truong MT, Kachnic LA, Grillone GA, Bohrs HK, Lee R, Sakai O, et al: Long-term results of conformal radiotherapy for progressive airway amyloidosis. Int J Radiat Oncol Biol Phys 83:734-739, 2012

\section{Disclosures}

The authors report no conflict of interest concerning the materials or methods used in this study or the findings specified in this paper.

\section{Author Contributions}

Drafting the article: Meier. Critically revising the article: all authors. Reviewed submitted version of manuscript: all authors. Approved the final version of the manuscript on behalf of all authors: Meier.

\section{Correspondence}

Teresa Meier, Department of Radiation Oncology, University of Cincinnati Medical Center, 234 Goodman St., ML 0757, Academic Health Center, PO Box 670757, Cincinnati, OH 45267-0757. email: meierta@ucmail.uc.edu. 